\title{
Dielectric and electromagnetic behavior of conductive nanocomposites polymers: PP/MWCNT investigations for EMI applications
}

\author{
Stefan Ursache ${ }^{1, a}$, Romeo Cristian Ciobanu ${ }^{1, b}$, Vlad Scarlatache ${ }^{1, c}$
} and Andrei Niagu ${ }^{1, d}$

\author{
1"Gheorghe Asachi" Technical University of lasi, Electrical Engineering Faculty, Bd. Profesor \\ Dimitrie Mangeron, nr. 21- 23, 700050, lasi, Romania
}

asursache@ee.tuiasi.ro(corresponding author), 'rciobanu@ee.tuiasi.ro, 'vscarlatache@ee.tuiasi.ro, daniagu@ee.tuiasi.ro

Keywords: polymer composites, conductive fillers, multi-walled carbon nanotubes, permittivity, dielectric losses, computer simulation, shielding.

\begin{abstract}
The paper highlights the most important dielectric features for some nanocomposites polymer matrix based on polypropylene (PP) with insertion of carbon nanotubes multi-walled (MWCNTs). The dielectric characteristics analyzed are the real permittivity and dielectric losses of the sample based on PP with 5\% insertion of MWCNTs. The measurements are made in a range of frequency between $1 \mathrm{MHz}$ to $3 \mathrm{GHz}$. The composite form was also analyzed through computer modeling and simulation and electromagnetic properties for EMC shielding applications are also considered. PP/MWCNTs composite with shielding effectiveness of 15-20 dB was investigated through modeling and simulation at about 5\% MWCNTs filling. Shielding mechanism was estimated by calculating the total shielding effectiveness (SE) into absorption and reflection loss. PP/MWCNTs composite indicates a shielding mostly by absorption mechanism; therefore it also can be used in other microwave applications or like a radar absorbing material. The effect of MWCNTs affects the electrical conductivity of the nanocomposite. The proposed material shows some interesting electromagnetic compatibility (EMC) properties and promises better performance using different amounts of MWCNT's.
\end{abstract}

\section{Introduction}

Electromagnetic interference (EMI) shielding is an unwelcome electromagnetic (EM) induction caused by wide use of alternative current/voltage and produces accidentally induced signals (voltage and current) in the vicinity of the electronic circuits, thereby trying to fault its performance. The mutual interference among electronic equipment, electric machines, process equipment or measurement instruments and result in disruption or complete failure of the normal appliances. Electromagnetic shielding is commonly used to reduce emissions and to increase immunity of different electronic equipment or in the area of protective panels for locative, working, medical spaces against high power EM emitters. Electromagnetic interference and Electromagnetic Compatibility (EMC) are becoming important issues in the design of novel high-speed transmission or GSM networks and the market for finding safe solutions and sure equipment, especially at network base-sites, has a fast growing with more than 5-7\% per year.

Consequently, some shielding mechanisms must be intended for ensure normal working of equipment regardless of the presence of external electromagnetic unwanted sources. For efficient shielding action, the screen (shield) should possess either mobile charge carriers (electrons or holes) or electric and/or magnetic dipoles which interact with the electric $(\mathrm{E})$ and magnetic $(\mathrm{H})$ vectors of the incident EM radiation. The designing of an EMI screen with a desired level of effectiveness, meeting a set of physical criteria, maintaining economics limits and regulating the involved shielding mechanism is not a straight forward task and also involves complex interaction of inherent characteristics (dielectric properties) of shielding material and logical selection of extrinsic parameters. Therefore, to touch the theoretically desired shielding performance of a materials and to 
satisfy complex design criteria, elementary knowledge of shielding theory, set of governing theoretical equations, important design parameters and relevant measurement technique must be studied with carefully [1].

Classical cellular solids used in shields/absorbers and Salisbury screen applications are either massive metallic or metal-multilayer structures. These material solutions do not offer enough degrees of freedom to the EM designer especially at $\mathrm{GHz}$ frequency domain, neither do they offer optimised configuration for the structural loading or thermal effects they have to bear. As we know, any enclosure shielding integrity is compromised by the presence of holes and apertures for thermal dissipation and for other technical purposes. If currents that following on conducting surfaces of a shielding structure, cross these apertures, they turn into unwanted antennas. In order to remove this circumstance is to use absorbing materials with dielectric EMC performances for projecting and designing shielding systems and structures. Nanocomposite dielectric materials containing conductive inclusions assigned randomly, including carbon nanotubes, at lower or higher concentrations can be used for shielding electromagnetic waves at RF and microwave frequencies [2]. Nonetheless, the request to reduce the weight and the cost of these „safety" structures requires the replacement of metal by novel materials like conducting polymers and composites. These composites may include metal insertions, carbon, chemical substances or other inserted components with good dielectric conductivity [3].

In this paper, there are illustrated the dielectric characteristics and electromagnetic behavior of a nanocomposite polymer matrix based on polypropylene (PP) with insertion of multiwall carbon nanotubes (MWCNTs).

\section{Materials and methods}

Polypropylene (PP) is a thermoplastic polyolefin with large scattered in products made by injection molding technique. PP is a semi-crystalline polymer that is extensively used due to its good thermic and plastic modeling properties, cost and for the easy processing [5].

Manufacturing techniques that polypropylene can be processed are varied: injection molding, blow molding, extrusion, multifilament, micro-oven or different profile extrusion [4]. Today is a great interest to develop new materials with polymer base matrix in composite form with different inclusions in order to improve physical, mechanical or chemical properties. This paper purpose a composite material that consist in a mixture of polypropylene with multi-walled carbon nanotubes, which would result in a material with better mechanical and physical properties. These properties can be improved by varying the percentage of the conductive inclusions (MWCNT's) in the base polymer (PP) matrix $[1,5]$.

A lot of studies were made on this nanocomposite material types in general for developing mechanical and electrical properties [5-7].

Because of the very low conductivity of the insulating polymer, a model that has any conduction of charge by the polymer would have conductivity not very different from the base polymer; hence, nanotubes must carry all the charge for high conductivities to be achieved. If nanotubes are in contact, then transfer of charge from one nanotube to another will offer much higher resistance than charge traveling along nanotubes, even for the case of semiconducting tubes. High resistances occur because of the limited contact area between two tubes. This type of resistance, termed constriction resistance, has been shown to be limiting because of increasing compression forces or decreasing the hardness of the filler leads to higher conductivities; constriction resistance is the limiting resistance [7]. The percolation threshold of multi-walled carbon nanotubes in polypropylene matrix depend on the processing behavior to obtain nanocomposition of these two materials. 


\section{Processing}

The CNTs used in this study, known under their commercial name as NC7000, are high conductivity MWCNTs, characterized by a mean diameter of $9.5-9.6 \mathrm{~nm}$, an average length of 1.5 $\mu \mathrm{m}$, a purity of carbon $90 \%$ and area of $250-300 \mathrm{~m}^{2} / \mathrm{g}$, products manufactured by Nanocyl S.A. The PP/MWCNTs nanocomposites were prepared separately by melt compounding PP pellets with $5 \%$ MWCNTs powders. The melt compounding was carried at $175{ }^{\circ} \mathrm{C}$ for 8 minutes, while the temperature was maintained at $50{ }^{\circ} \mathrm{C}$. The samples was performed by direct melt compounding and have a diameter of $5 \mathrm{~mm}$ and a thickness of $2.9 \mathrm{~mm}$. Spherical sample with appropriate dimensions for measuring cell was extracted from material with a specialized punch.

\section{Dielectric analysis}

\section{Investigation method}

In the measuring process of dielectric parameters has been used Agilent E4991A impedance analyzer and a dielectric cell BDS 2200 that was connected using the extension 002 of the broadband spectrometer Novocontrol, concept 80. The BDS2200 RF sample cells are designed for dielectric properties measurements in $1 \ldots 3 \mathrm{GHz}$ frequency range. Also for temperature variation was used a specialized thermal controller named Quatro Cryosystem. With Agilent E4991A impedance analyzer can be measured both the magnetic and dielectric characteristics of the materials, but with different cells. In this paper we will focus on measuring the dielectric characteristics with the system presented in Fig. 1.

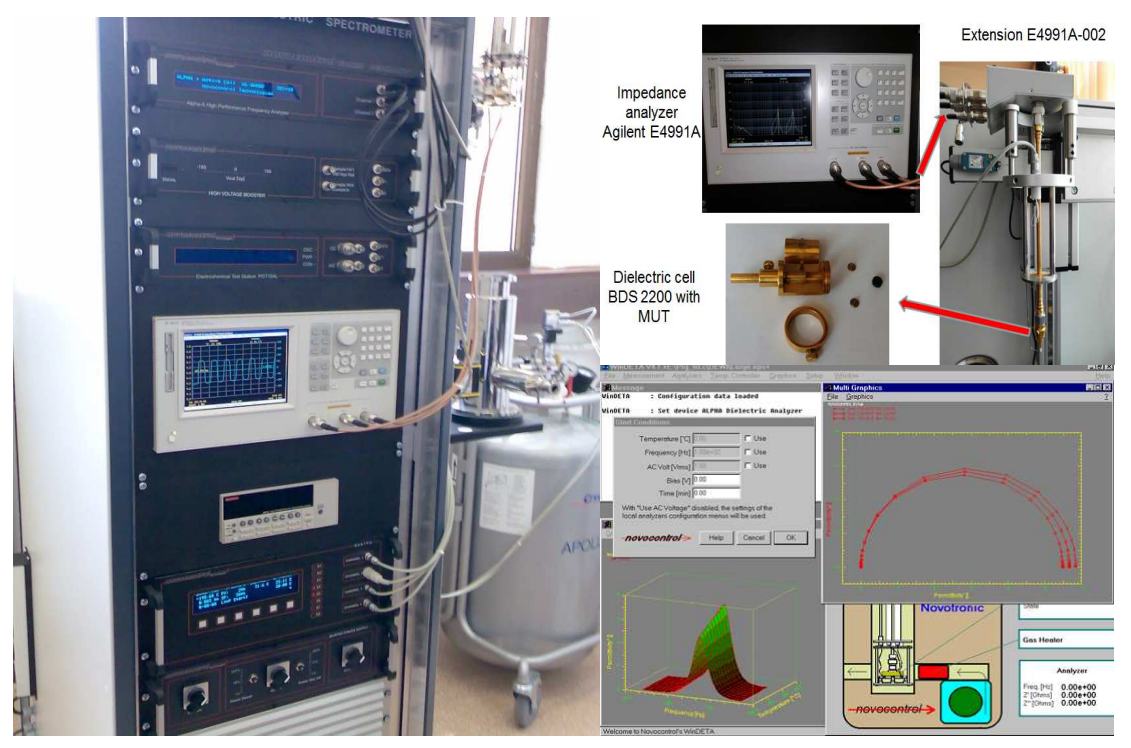

Fig. 1. The measurement system of the dielectric characteristics at high frequency

The dielectric properties of the samples were analyzed on the basis of the complex permittivity, $\varepsilon^{*}(\omega)[8,9]$.

Mathematically, it can be represented as a combination of the real part, $\varepsilon^{\prime}(\omega)$, and the imaginary part, $\varepsilon^{\prime \prime}(\omega)$ [equation: $\varepsilon^{*}(\omega)=\varepsilon^{\prime}(\omega)-\mathrm{i} \varepsilon^{\prime \prime}(\omega)$.

The peak in imaginary permittivity and step change in real permittivity, which represents the relaxation, can be described mathematically by the Havriliak-Negami $(\mathrm{HN})$ equation:

$$
\varepsilon^{*}(\omega)=\frac{\Delta \varepsilon}{\left\{1+(i \omega \tau)^{1-\alpha}\right\}}+\varepsilon_{\infty}
$$


where $\Delta \varepsilon$ (the dielectric strength) and $\tau$ (the relaxation time constant) describe the strength and time dependence of the relaxation process respectively and the quantity $\varepsilon_{\infty}$ is the real permittivity at frequencies much higher than the relaxation process (i.e. the instantaneous behaviour). $\alpha$ and $\beta$ in Eq. (1) represent the $\mathrm{HN}$-shape parameters.

The study of real and imaginary part of permittivity can highlight very easy the evolution of polarization mechanisms of a material exposed to an electric field. Four different polarization mechanisms are discussed by the scientific literature [8]:

- Rotational or Dipolar

- Electronic

- Ionic

- Interfacial or Maxwell-Wagner.

The dielectric behavior [9] can be analyzed based on Debye-equation (2). The Cole-Cole plot or Argand plot displays dielectric spectra as $\varepsilon$ '' $(\omega)$ versus $\varepsilon$ '' $(\omega)$ and can be helpful for representing the relaxations in polymers.

$$
\varepsilon^{*}(\omega)-\varepsilon_{\infty}=\frac{\varepsilon_{S}-\varepsilon_{\infty}}{1+(i \omega \tau)^{1-\alpha}}
$$

where $\varepsilon_{\mathrm{s}}$ and $\varepsilon_{\infty}$ are the "static" and "infinite frequency" dielectric constants.

\section{Broadband dielectric spectroscopy results}

The measurements were performed in the frequency band: $1 \mathrm{MHz} \div 3 \mathrm{GHz}$ in 20 points with a logarithmic scale. The temperature range for tests was $30 \div 120{ }^{\circ} \mathrm{C}$, with an increment of $5{ }^{\circ} \mathrm{C}$.

Sample used for measurements in this frequency range have a thickness of $2.9 \mathrm{~mm}$. The sample is prepared between two gold plated copper electrodes forming a sample capacitor with the material under test as dielectric layer. The Graphical representation is made in $2 \mathrm{D}$ and $3 \mathrm{D}$ form for the clarity of the results.

The 2D and 3D representation of the real permittivity for sample PP-MWCNT at high frequency depend on temperature variation are presented in Fig 2. A slight relaxation can be observed at around frequencies of $500 \mathrm{MHz}$, due to ionic polarization mechanism, that is specific to this frequency band, but that is not specific to these types of materials [9].
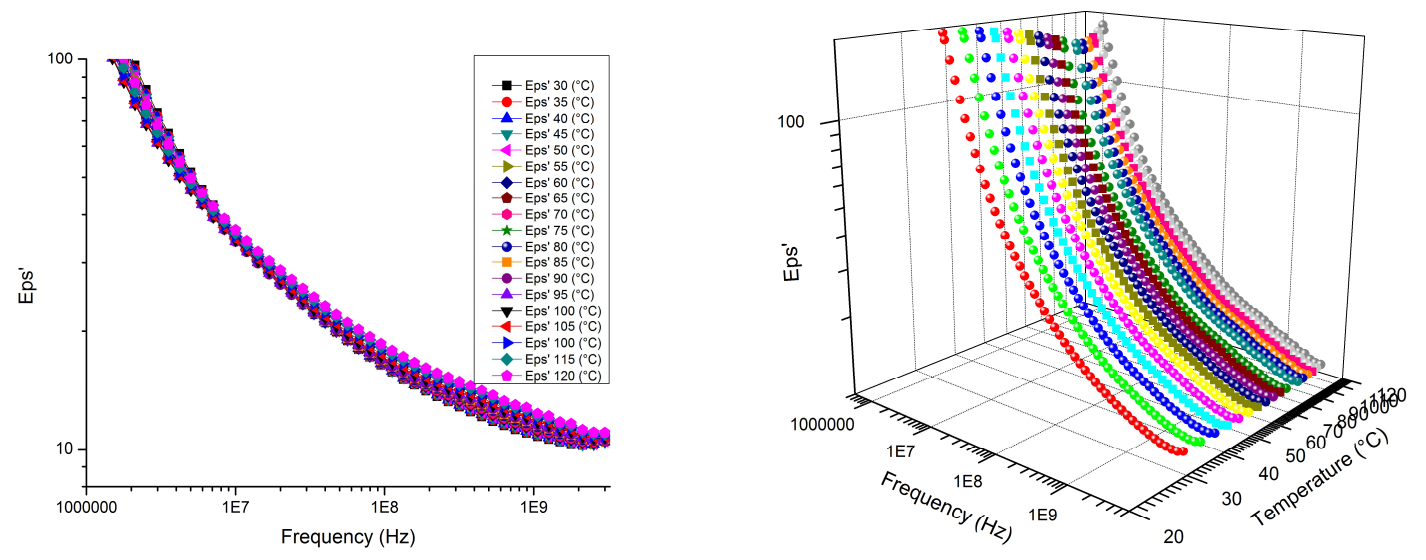

Fig. 2. The 2D and 3D diagram of real permittivity for sample PP-MWCNT at HF depending on the temperature variation 

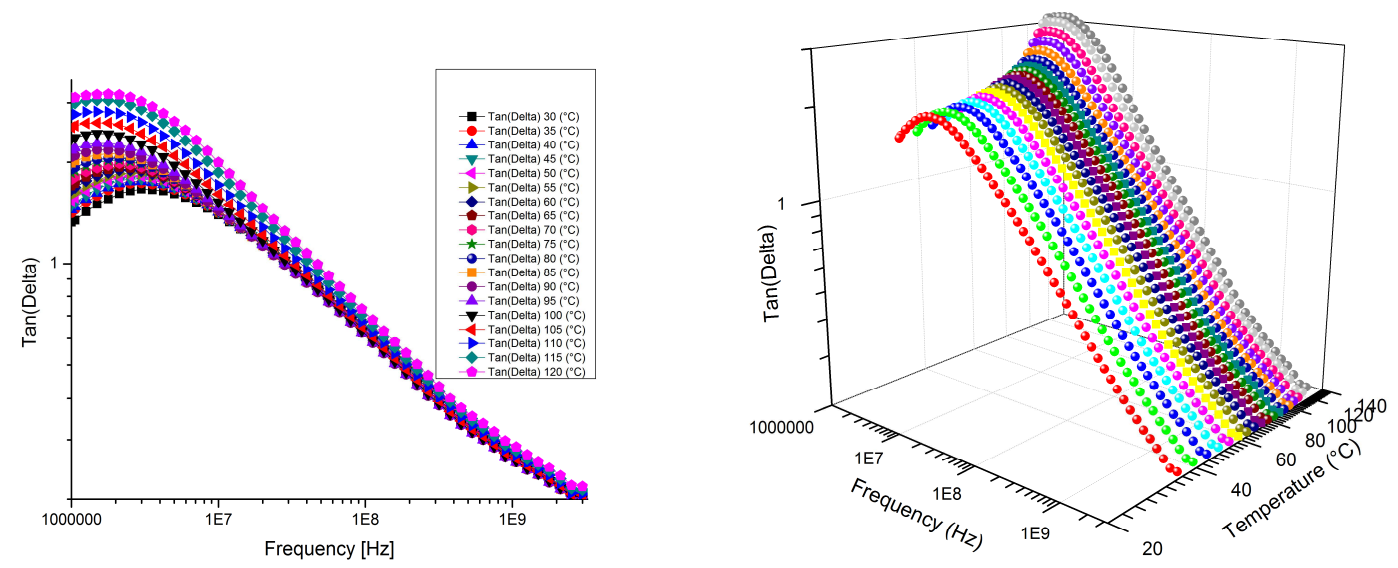

Fig. 3. The 2D and 3D diagram of the loss angle tangent for sample PP-MWCNT at HF depending on the temperature variation

The evolution of the dielectric losses in $2 \mathrm{D}$ and 3D representation is shown in Fig. 3, as Tan (Delta), where a peak is observed clearly around the frequency of a few $\mathrm{MHz}$, a phenomenon that is due to the dipolar polarization or polarization of orientation, which was observed more clearly in the material analysis at low frequency [9]. This peak becomes more noticeable with increasing the temperature and disappears with increasing of frequency. Also a step is observed along the reduction of Tan (Delta) around the frequency of $500 \mathrm{MHz}$, due to the light influence of ionic polarization.

\section{Electromagnetic behavior analysis}

Generally, EMC simulations software numerically treats the Maxwell's equations and their evolution depending on material properties and electromagnetic field on a computer. In this paper, the results were obtained and the graphs were plotted in transient solver. In contrast to the frequency solver used by others simulators the transient approach can cover wider broadband frequency behaviour of the simulated structure from only one calculation run. This solver is suitable and efficient for most kinds of high frequency applications such as radars absorbers, waveguides, filters, most types of antennas and many other electromagnetic approaches. Multilevel Subgridding Scheme (MSS ${ }^{\mathrm{TM}}$ ), integrated module in this solver is intended to improve the meshing efficiency and to speed up simulations for difficult and complex structures [11].

The shielding efficiency (SE) is generally expressed and quantified within the meaning of reduction in amplitude of incident power/field upon transition across the material/screen - Fig. 4 and Fig. 5.

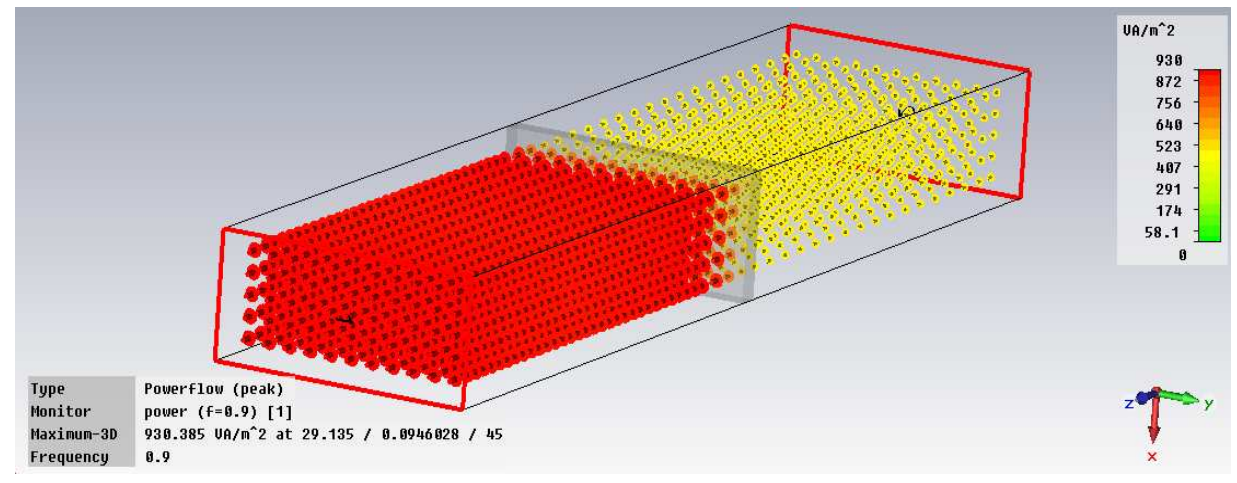

Fig. 4. Power flow reduction by the composite shield at $0.9 \mathrm{GHz}$ 


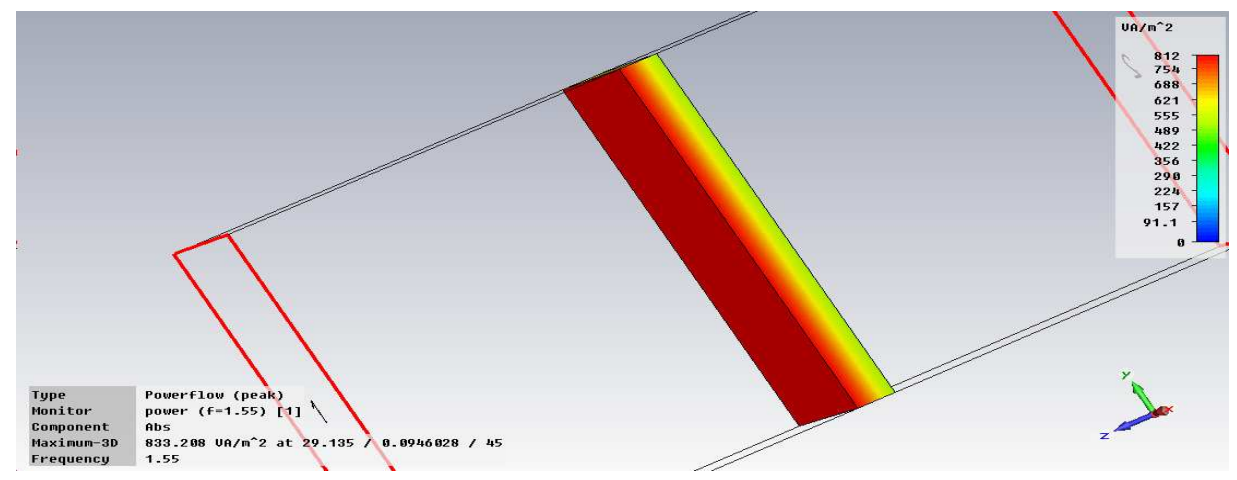

Fig. 5. Power flow reduction in the material at $1.5 \mathrm{GHz}$

Electromagnetic simulation results are expressed as the $\mathrm{S}$ (scattering) parameters for the structure. In the electromagnetic shielding theory the typical macroscopic parameters that define the reflectance, transmittance and absorption are defined with reference to the electromagnetic power flow - Fig. 6 and are linked to the S parameters [10] as presented in equations (3-5):

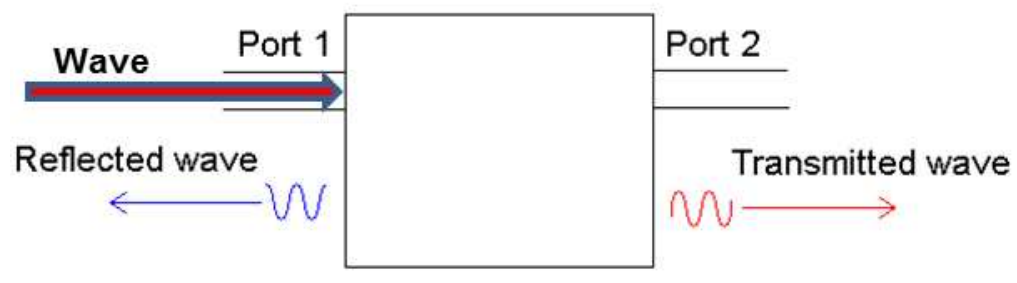

Fig. 6. Schematic representation of S parameters

$$
\begin{gathered}
R=\left|S_{11}\right|^{2} \\
T=\left|S_{21}\right|^{2} \\
A=1-R-T=1-\left|S_{11}\right|^{2}-\left|S_{21}\right|^{2}
\end{gathered}
$$

The structure under numeric tests was designed with dimensions $40 \mathrm{~mm}$ x $20 \mathrm{~mm}$ x $4.5 \mathrm{~mm}$ and with dielectric characteristics previously obtained. The values for electric permittivity and loss angle tangent were parametric varied corresponding to different temperatures at which the sample was tested.

The investigations were performed at the interaction between a plane wave and the composite structure under test with material's parameters previously taken, at normal incidence. The boundary conditions are considered to electric wall (both walls on $\mathrm{x}$ directions) and magnetic wall (y direction walls). At some space from the designed sample an input wave port is placed; the second (exit) wave port is added only when losses inside the structure are taken into account, Fig. 6.

The numeric tests of the electromagnetic behaviour for the composite were made using parametric sweep option of the CST software for values corresponding on dielectric permittivity $(\varepsilon)$ and loss tangent $(\operatorname{tg} \delta$ ) previously obtained with broadband dielectric spectroscopy.

The frequency range for de solver was considered in the range 0.1 to $3 \mathrm{GHz}$ because it comprise the most used frequencies in the civil mobile communications (GSM, UMTS, Wi-Fi, Bluetooth), one of the target application of the proposed composite.

The results are plotted in Fig. 7 and Fig. 8. Fig. 7 shows the electromagnetic reflective properties of the material defined with $\mathrm{S}_{11}$ parameter. In Fig. 8, the electromagnetic transmission of the wave through the structure is plotted defined by $\mathrm{S}_{21}$. Because of appropriate results the electromagnetic tests were made for values of dielectric characteristics (permittivity and loss tangent) corresponding on a few temperatures. 


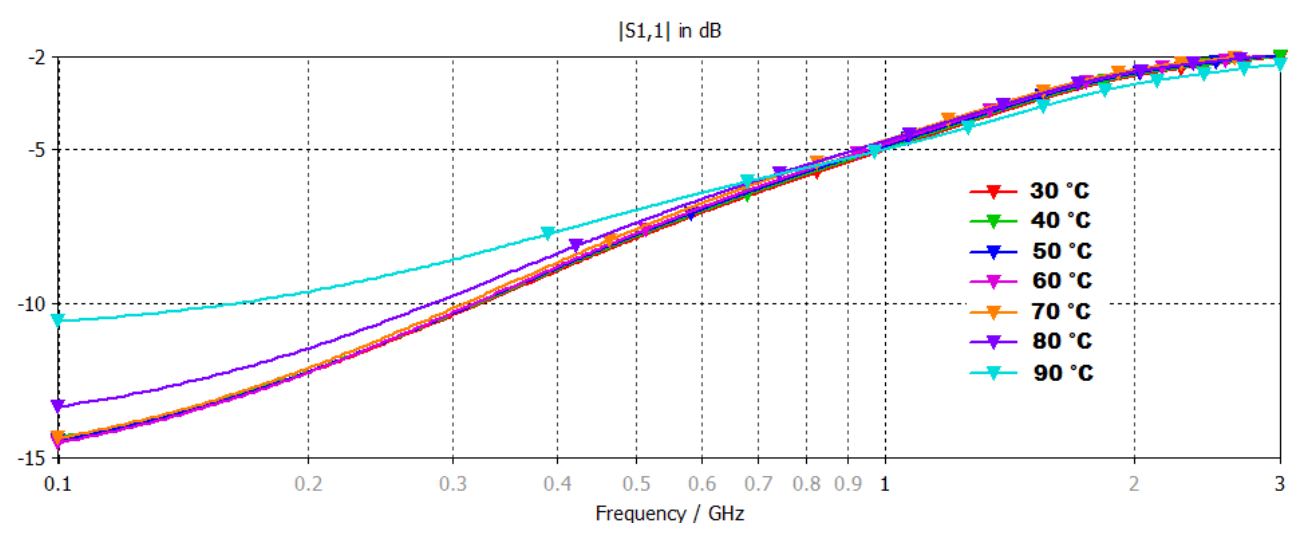

Fig. 7. Reflection at different temperatures vs. frequency

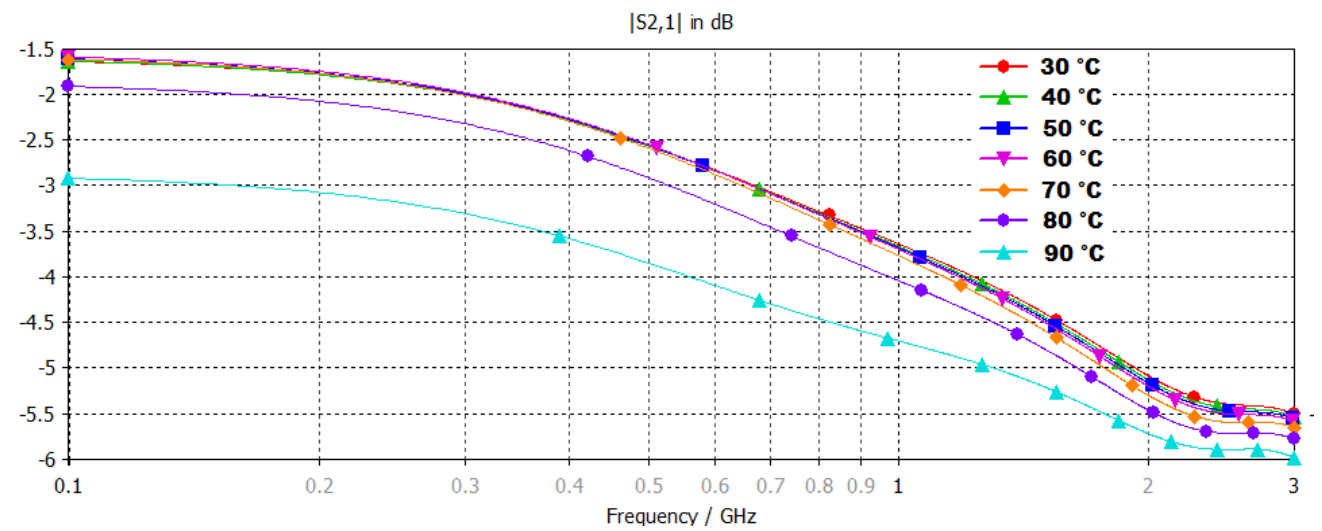

Fig. 7. Transmission vs. frequency

\section{Conclusions}

This paper presents the dielectric characteristics of a polymer nanocomposite material with insertion of multilayer nanotubes carbon. These materials have a very interesting evolution of the dielectric characteristics in frequency and temperature variation, due to the polarization mechanisms present. At frequencies in order of $\mathrm{MHz}$ appears the polarization of orientation that increase with increasing the temperature. Analyzing the dielectric characteristics at high-frequency, observe that mechanism of polarization is present at all temperatures. The electrical conduction of this material increases with increasing the frequency. If at low frequency this material has an insulating character, with increasing the frequency it has a semiconductor character.

The study of electromagnetic behavior show that the EMC performances are influenced mainly by the conductivity (related to loss tangent) of the dielectric, which means that the growing percent of MWCNT will expect better results [12]. These parameters are also influenced by the temperature variation, so it is better to take into account this information in applications with this kind of materials or in in future research.

In scope to predict the behavior of electromagnetic phenomena, that are highly complex physical process we use numerical techniques to simulate and after that to make a model as close to reality of this. We also use real measurements techniques like dielectric spectroscopy which is a very complex method for materials and fields characterization for validate the numerical model obtained. The selection of any numerical solver or technique requires good knowledge of its basic formulation and of course the input variables for correctly applying appropriate technique for the problem or phenomena under analysis. 


\section{Acknowledgment}

This paper was supported by the project "Development and support of multidisciplinary postdoctoral programmes in major technical areas of national strategy of Research - Development Innovation" 4D-POSTDOC, contract no. POSDRU/89/1.5/S/52603, project co-funded by the European Social Fund through Sectoral Operational Programme Human Resources Development 2007-2013 and also by the project POSDRU CUANTUMDOC "Doctoral studies for European performances in research and innovation", (ID79407) financed by the European Social Fund and the Romanian Government.

\section{References}

[1] P. Saini and M. Arora, Microwave Absorption and EMI Shielding Behavior of Nanocomposites Based on Intrinsically Conducting Polymers, Graphene and Carbon Nanotubes, New Polymers for Special Applications, Ailton De Souza Gomes (Ed.), ISBN: 978-953-51-0744-6, InTech 2012, doi: 10.5772/48779.

[2] Y. Li, J. Zhu, S. Wei, J. Ryu, Q. Wang, L. Sun and Z. Guo, Poly(propylene) Nanocomposites Containing Various Carbon Nanostructures, Macromol. Chem.Phys., Vol. 212, No. 22, (2011) 2429-2438.

[3] M. Y. Koledintseva, J. L. Drewniak, R. E. DuBroff, K. N. Rozanov, and B. Archambeault, Modeling of shielding composite materials and structures for microwave frequencies, Progress In Electromagnetics Research B, Vol. 15 (2009) 197-215 doi: 10.2528/PIERB09050410

[4] S. Lee, M. W. Kim, S. H. Kim, Rheological and electrical properties of polypropylene/MWCNT composites prepared with MWCNT masterbatch chips, European Polymer Journal, 44(2008) 1620-1630.

[5] Y. Pan, H. K. F Cheng, L. Li, S. H. Chan, a.a, Annealing induced electrical conductivity jump of multi-walled carbon nanotube/polypropylene composites and influence of molecular weight of polypropylene. J. Polym. Sci. B Polym. Phys., 48 (2010): 2238-2247. doi: 10.1002/polb.22106

[6] A. Chiolerio, M.Castellino, P. Jagdale, M. Giorcelli, S. Bianco and A. Tagliaferro, Electrical Properties of CNT-Based Polymeric Matrix Nanocomposites, Carbon Nanotubes - Polymer Nanocomposites, Chapter 11, InTech 2011, doi: 10.5772/18900

[7] A. E. Daugaard, Katja Jankova, J.M.R Marín, J. Bøgelund, S. Hvilsted, Poly(ethylene-cobutylene) functionalized multi walled carbon nanotubes applied in polypropylene nanocomposites, European Polymer Journal, 48, 4, pp. 743-750, (2012).

[8] F. Kremer, A. Schonhals, Broadband Dielectric Spectroscopy, Springer, 2003.

[9] M. Olariu, V. A. Scarlatache, A. Niagu, S. Ursache, R. C. Ciobanu, The Influence of Frequency and Temperature upon Dielectric Behavior of Polypropylene reinforced with Multi-walled Carbon Nanotubes (MWCNTs), Proceedings of the 13th International Conference on Optimization of Electrical and Electronic Equipment, Brasov 2012.

[10] R. F. Damian, R. Ciobanu, C. Schreiner, EMC tests and properties vs. microstructure for auxetic materials", 15th IMEKO TC4 Symposium on Novelties in Electrical Measurements and Instrumentation, Iasi, Romania, 2007.

[11] www.cst.de

[12] Junhua Wu, D.D.L. Chung, Combined Use of Magnetic and Electrically Conductive Fillers in a Polymer Matrix for Electromagnetic Interference Shielding, Journal of Electronic Materials August 2008, Volume 37, Issue 8, Springer 1088-1094, doi:10.1007/s11664-008-0486-4 